\title{
Nonclassicality and decoherence of photon-subtracted squeezed states
}

\author{
Asoka Biswas $^{1}$ and G. S. Agarwal ${ }^{2}$ \\ ${ }^{1}$ Department of Chemistry, University of Southern California, Los Angeles, California 90089, USA \\ ${ }^{2}$ Department of Physics, Oklahoma State University, Stillwater, Oklahoma 74078, USA
}

(Received 4 December 2006; published 7 March 2007)

\begin{abstract}
We discuss nonclassical properties of single-photon subtracted squeezed vacuum states in terms of the sub-Poissonian statistics and the negativity of the Wigner function. We derive a compact expression for the Wigner function from which we find the region of phase space where Wigner function is negative. We find an upper bound on the squeezing parameter for the state to exhibit sub-Poissonian statistics. We then study the effect of decoherence on the single-photon subtracted squeezed states. We present results for two different models of decoherence, viz. amplitude decay model and the phase diffusion model. In each case we give analytical results for the time evolution of the state. We discuss the loss of nonclassicality as a result of decoherence. We show through the study of their phase-space properties how these states decay to vacuum due to the decay of photons. We show that phase damping leads to very slow decoherence, slower than the photon-number decay.
\end{abstract}

DOI: 10.1103/PhysRevA.75.032104

PACS number(s): 03.65.Yz, 42.50.Dv

\section{INTRODUCTION}

Quantum states can be well-described in terms of Wigner functions. The states with Gaussian Wigner function have been of particular interest in the context of quantum information processing. Entanglement in such states in terms of quadratures is also well-studied. On the other hand, the quantum states with non-Gaussian Wigner function are also quite important. For example, a single-photon state, which finds many applications in quantum information processing, shows non-Gaussian behavior in phase space. In a recent experiment, a non-Gaussian state has been produced by a homodyne detection technique from a single-mode squeezed state of light $[1,2]$. For certain non-Gaussian states, Wigner functions can take negative values. Such negativity refers to nonclassicality of these states. These states are useful in entanglement distillation [3,4], loophole-free tests of Bell's inequality [5], and quantum computing [6]. A specific class of such nonclassical states has been shown to be similar to the Schrodinger kitten state, in the sense that their Wigner functions show negativity at the origin of phase space $[7,8]$. It is well-known that the Schrodinger cat states [9], which are quantum superpositions of coherent states, are nonclassical in nature and are very important to study the interface of quantum and classical worlds. Superposition of coherent states with low amplitudes creates Schrodinger cat-like states known as Schrodinger kitten states. Most of the experiments to prepare the Schrodinger cat states have been performed in cavities or bound systems. Thus they are not much useful in quantum information networks though they have the nonGaussian nature which is required in certain quantum communication protocols. In $[7,8]$, it has been shown how to prepare a Schrodinger kitten state in an optical system by subtracting a single photon from a squeezed vacuum state. This optical kitten state would overcome the limitations of bound systems. Repeated photon-subtractions can lead to conditional generation of an arbitrary single-mode state [10]. We note that similar non-Gaussian states could be prepared by adding a single photon to a squeezed vacuum state (see
$[11,12]$ for details of photon-added coherent states, which are also non-Gaussian states). These states are equivalent to a single-photon subtracted squeezed vacuum state and exhibit similar behavior in phase space. It is worth mentioning that non-Gaussian two-mode entangled states can be prepared by subtracting a photon from a two-mode squeezed state $[13,14]$.

In this paper, we focus our study on the nonclassical properties and decoherence of single-photon subtracted squeezed vacuum states which are optically produced single-mode non-Gaussian states. The structure of the paper is as follows. In Sec. II, we introduce the photon-subtracted squeezed state and discuss its nonclassical properties in terms of the subPoissonian statistics and the negativity of its Wigner function. We derive a compact expression of the Wigner function and find the region in phase space where it becomes negative. We show that there is an upper bound of the squeezing parameter for this state to exhibit sub-Poissonian statistics. In Sec. III, we study the effects of two different models of decoherence: photon-number decay and phase damping. In both cases, we derive analytical expressions for the timeevolution of the state and its Wigner function. We discuss the loss of nonclassicality due to decoherence. We show through the study of evolution of the Wigner function how the state decays to vacuum as a result of photon-number decay. We further show that phase damping leads to much slower decoherence than the photon-number decay.

\section{PHOTON-SUBTRACTED SQUEEZED STATES}

An un-normalized single-mode squeezed vacuum state is given by

$$
|\psi\rangle \equiv S(\zeta)|0\rangle, \quad S(\zeta)=\exp \left[\frac{\zeta}{2} a^{\dagger 2}-\frac{\zeta^{*}}{2} a^{2}\right],
$$

where $S(\zeta)$ is the squeezing operator, $\zeta=r e^{i \theta}$ is the complex squeezing parameter, and $a$ is the annihilation operator. The Wigner function of this state is Gaussian and positive in 
(a)

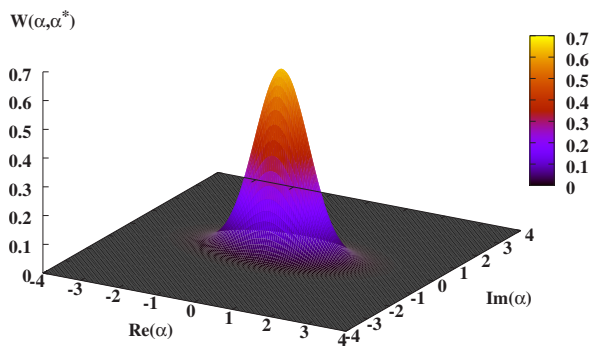

(b)

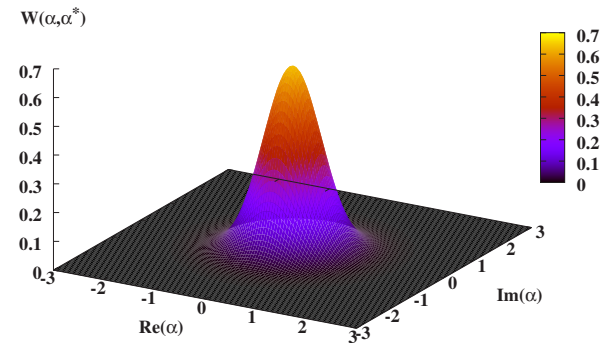

FIG. 1. (Color online) (a) Gaussian Wigner function $W_{\text {sq }}$ of the squeezed vacuum state $|\psi\rangle$ for $\theta=0$ and $r=0.31$. (b) The Gaussian Wigner function of a vacuum state. phase space. When $p(>0)$ number of photons are subtracted from such states, the state can be written as

$$
|\psi\rangle_{p} \equiv a^{p} S(\zeta)|0\rangle \equiv a^{p} \exp \left[\frac{\xi}{2} a^{\dagger 2}\right]|0\rangle,
$$

where $\xi=\tanh (\zeta)$. For odd $p=2 m+1$, the normalized form of this state can be written as

$$
|\psi\rangle_{p}=\frac{1}{N_{o}} \sum_{s=0}^{\infty} \frac{(\xi / 2)^{s+m+1}}{(s+m+1) !} \frac{(2 s+2 m+2) !}{\sqrt{2 s+1}}|2 s+1\rangle,
$$

while for even $p=2 m$, the state becomes

$$
|\psi\rangle_{p}=\frac{1}{N_{e}} \sum_{s=0}^{\infty} \frac{(\xi / 2)^{s+m}}{(s+m) !} \frac{(2 s+2 m) !}{\sqrt{2 s}}|2 s\rangle,
$$

where $N_{o}$ and $N_{e}$ are the normalization constants. In this paper, we focus on the case when a single photon is subtracted from the squeezed vacuum, i.e., for $m=0$ in Eq. (3).

\section{A. Negativity of the Wigner function}

The Wigner function of the squeezed vacuum state $|\psi\rangle$ is given by

$$
W_{\mathrm{sq}}\left(\alpha, \alpha^{*}\right)=\frac{2}{\pi} \exp \left(-2|\widetilde{\alpha}|^{2}\right)
$$

where $\tilde{\alpha}=\alpha \cosh (r)-\alpha^{*} e^{i \theta} \sinh (r)$. The function is Gaussian in phase space, as shown in Fig. 1. We now calculate the Wigner function of the state $|\psi\rangle_{p}$. This state can be rewritten as

$$
|\psi\rangle_{p}=a^{p} S(\zeta)|0\rangle=S(\zeta) S^{\dagger}(\zeta) a^{p} S(\zeta)|0\rangle .
$$

Using the relation

$$
S^{\dagger}(\zeta) a^{p} S(\zeta)=\left[\cosh (r) a+e^{i \theta} \sinh (r) a^{\dagger}\right]^{p},
$$

we get the following from Eq. (6):

$$
|\psi\rangle_{1} \equiv S(\zeta)|1\rangle
$$

for $p=1$. For a density matrix $\tilde{\rho}\left(a, a^{\dagger}\right)$, we can write the following:

$$
S(\zeta) \widetilde{\rho}\left(a, a^{\dagger}\right) S^{\dagger}(\zeta)=\widetilde{\rho}\left[S(\zeta) a S^{\dagger}(\zeta), S(\zeta) a^{\dagger} S^{\dagger}(\zeta)\right] .
$$

We write the Wigner function of $\widetilde{\rho}\left(a, a^{\dagger}\right)$ as $W_{\tilde{\rho}}\left(\alpha, \alpha^{*}\right)$. Using the identities

$$
\begin{gathered}
S(\zeta) a S^{\dagger}(\zeta)=a \cosh (r)-a^{\dagger} e^{i \theta} \sinh (r), \\
S(\zeta) a^{\dagger} S^{\dagger}(\zeta)=a^{\dagger} \cosh (r)-a e^{-i \theta} \sinh (r),
\end{gathered}
$$

in Eq. (9), we can thus write the Wigner function of the density matrix $\rho\left(a, a^{\dagger}\right)=S(\zeta) \widetilde{\rho}\left(a, a^{\dagger}\right) S^{\dagger}(\zeta)$ as

$$
W_{\rho}\left(\alpha, \alpha^{*}\right)=W_{\widetilde{\rho}}\left(\tilde{\alpha}, \widetilde{\alpha}^{*}\right),
$$

where we have used the linearity property of the Wigner function. For the state (8), $\tilde{\rho}=|1\rangle\langle 1|$ and its Wigner function is given by

$$
W_{\widetilde{\rho}}\left(\alpha, \alpha^{*}\right)=\frac{2}{\pi}\left(4|\alpha|^{2}-1\right) e^{-2|\alpha|^{2}} .
$$

Thus the Wigner function of the single-photon subtracted squeezed vacuum state becomes

$$
W_{\rho}\left(\alpha, \alpha^{*}\right)=\frac{2}{\pi}\left(4|\widetilde{\alpha}|^{2}-1\right) e^{-2|\tilde{\alpha}|^{2}},
$$

where we have used Eqs. (12) and (13). Clearly, the Wigner function (14) is non-Gaussian in phase space. We show the plot of this Wigner function in the phase space in Fig. 2 for different squeezing parameters. As an evidence of nonclassicality of the state, squeezing in one of the quadratures is clear in the plots. Also there is some negative region of the Wigner function in the phase space which is another evidence of the nonclassicality of the state. The function becomes negative in phase space, when
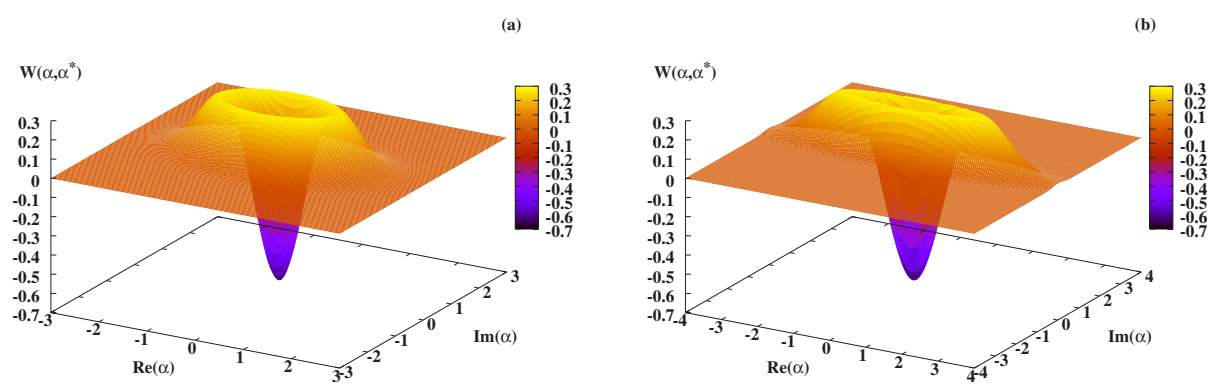

FIG. 2. (Color online) Plots of Wigner functions of single-photon subtracted squeezed states for (a) $r=0.31$ and (b) $r=0.8$ with $\theta=0$. 


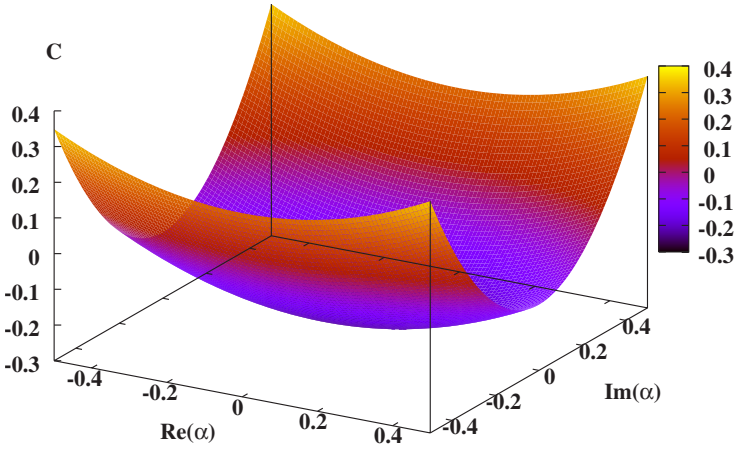

FIG. 3. (Color online) Variation of $C$ in phase space for $r$ $=0.31$ and $\theta=0$.

$$
|\widetilde{\alpha}|^{2}<\frac{1}{4}
$$

We show in Fig. 3 the variation of $C=|\widetilde{\alpha}|^{2}-\frac{1}{4}$ in phase space. The negative region of $C$ corresponds to the negativity of the Wigner function. Note that $C=$ const corresponds to ellipse in phase space, as shown in Fig. 4.

Note that the photon-subtracted squeezed states are similar to Schrodinger kitten states $[7,8]$ because Wigner functions exhibit the same characteristics in phase space especially for the large values of the squeezing parameters. Moreover, in both cases, Wigner function becomes negative in the center of phase space.

\section{B. Sub-Poissonian nature of the photon-subtracted state}

The nonclassicality of the state $|\psi\rangle_{1}$ can also be analyzed by studying its sub-Poissonian character in terms of Mandel's $Q$-parameter [15] which is defined by

$$
Q=\frac{\left\langle a^{\dagger 2} a^{2}\right\rangle-\left\langle a^{\dagger} a\right\rangle^{2}}{\left\langle a^{\dagger} a\right\rangle} \text {. }
$$

The negativity of the $Q$-parameter refers to sub-Poissonian statistics of the state. However in [16], it has been shown that a state can be nonclassical even if $Q$ is positive. A similar situation occurs in the present case. For the state (3), we find

$$
\left\langle a^{\dagger 2} a^{2}\right\rangle=\sum_{n=0}^{\infty} n(n-1) \rho_{n, n}=\frac{3|\xi|^{4}\left(3+2|\xi|^{2}\right)}{N_{o}^{2}\left(1-|\xi|^{2}\right)^{7 / 2}}
$$
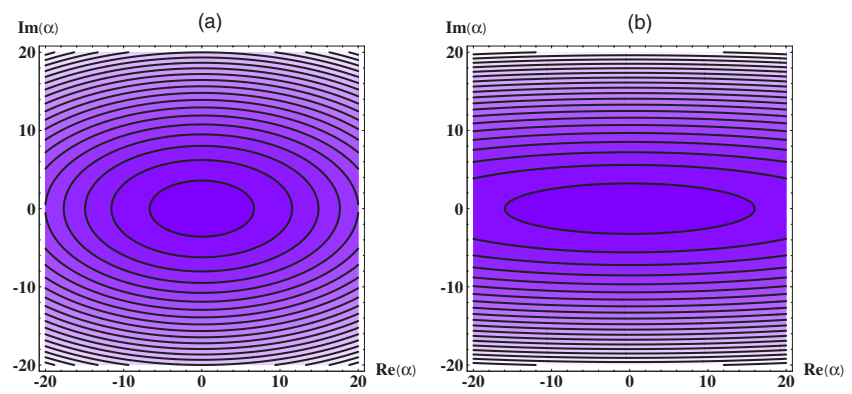

FIG. 4. (Color online) Contour plot for $C=$ const in phase space for (a) $r=0.31$ and (b) $r=0.8$.

$$
\left\langle a^{\dagger} a\right\rangle=\sum_{n=0}^{\infty} n \rho_{n, n}=\frac{|\xi|^{2}\left(1+2|\xi|^{2}\right)}{N_{o}^{2}\left(1-|\xi|^{2}\right)^{5 / 2}}
$$

where the normalization constant is given by

$$
N_{o}^{2}=\frac{|\xi|^{2}}{\left(1-|\xi|^{2}\right)^{3 / 2}} \text {. }
$$

From Eq. (17), we find that $Q$ becomes negative for $|\xi|$ $\lesssim 0.43$, which is satisfied for $r \leqq 0.46$. We emphasize that the Wigner function has a negative region for all values of $r$, and thus the photon-subtracted squeezed state is nonclassical for all $r$, though it does not exhibit sub-Poissonian photon statistics above a certain squeezing threshold.

\section{MODELS OF DECOHERENCE}

We next consider how this state evolves under decoherence. The decoherence of the single-mode state (3) can be due to decay of photons to the reservoir or due to phase damping.

\section{A. Amplitude decay model}

When the photons decay to reservoir, the corresponding Markovian dynamics of the state is well-described by the following equation:

$$
\frac{d}{d t} \rho=-\kappa\left(a^{\dagger} a \rho-2 a \rho a^{\dagger}+\rho a^{\dagger} a\right),
$$

where $\kappa$ is the rate of decay. The solution of this equation can be written as

$$
\rho(t)=\sum_{n, n^{\prime}} \rho_{n, n^{\prime}}(t)|n\rangle\left\langle n^{\prime}\right|,
$$

where the density matrix element $\rho_{n, n^{\prime}}(t)$ can be found by using the Laplace transformation and the iteration methods [17]. To see this, let us start with the time-dependent equation for $\rho_{n, n^{\prime}}$ :

$$
\dot{\rho}_{n, n^{\prime}}=-\kappa\left(n+n^{\prime}\right) \rho_{n, n^{\prime}}+2 \kappa \sqrt{(n+1)\left(n^{\prime}+1\right)} \rho_{n+1, n^{\prime}+1} .
$$

Using the new subscripts $q=n-n^{\prime}$ and $p=\left(n+n^{\prime}\right) / 2$, the above equation transforms into

$$
\dot{\rho}_{p, q}=-2 \kappa p \rho_{p, q}+2 \kappa \sqrt{(p+1)^{2}-(q / 2)^{2}} \rho_{p+1, q} .
$$

Taking the Laplace transformation of Eq. (22) and using the original subscript $n$ and $n^{\prime}$, we can write the time-dependent solution for the density matrix elements as

$$
\begin{aligned}
\rho_{n, n^{\prime}}(t)= & e^{-\kappa t\left(n+n^{\prime}\right)} \sum_{r=0}^{\infty} \sqrt{\left({ }^{n+r} C_{r}\right)\left({ }^{n^{\prime}+r} C_{r}\right)} \\
& \times\left(1-e^{-2 \kappa t}\right)^{r} \rho_{n+r, n^{\prime}+r}(t=0),
\end{aligned}
$$

where for the single-photon subtracted squeezed vacuum 


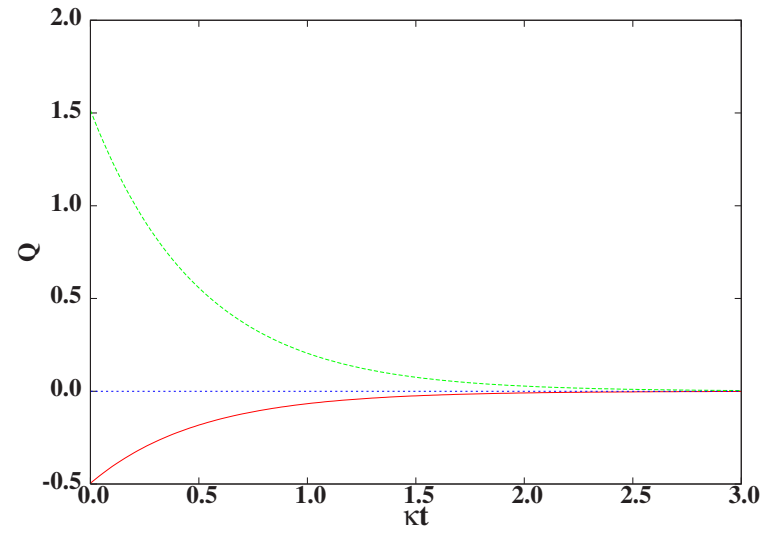

FIG. 5. (Color online) Variation of the $Q$-parameter with time in the presence of decoherence due to decay of photon for squeezing parameters $r=0.31$ (red line) and $r=0.8$ (green line).

$$
\begin{aligned}
\rho_{n+r, n^{\prime}+r}(t=0) & \left.=\frac{1}{N_{o}^{2}} \frac{(\xi / 2)^{(n+r+1) / 2}\left(\xi^{*} / 2\right)^{\left(n^{\prime}+r+1\right) / 2}}{2}\right) !\left(\frac{n+r+1}{2}\right) ! \\
& \times \frac{(n+r+1) !\left(n^{\prime}+r+1\right) !}{\sqrt{(n+r) !\left(n^{\prime}+r\right) !}} .
\end{aligned}
$$

We next calculate the parameter $Q$ [Eq. (16)] for the state (20). We have found that

$$
\begin{gathered}
\left\langle a^{\dagger 2} a^{2}\right\rangle=\sum_{n=0}^{\infty} n(n-1) \rho_{n, n}(t), \\
\left\langle a^{\dagger} a\right\rangle=\sum_{n=0}^{\infty} n \rho_{n, n}(t),
\end{gathered}
$$

where $\rho_{n, n}(t)$ is given by Eq. (23) and (24) for $n=n^{\prime}$ in case of state $|\psi\rangle_{1}$. Using Eqs. (25), we plot $Q$ with time in Fig. 5. It is easy to see that at long times $(\kappa t \rightarrow \infty), Q$ vanishes. This is because at this limit, $\rho_{n, n}(t)$ vanishes for all nonzero $n$ and $\rho_{0,0}(t \rightarrow \infty)=1$, i.e., the state decays to vacuum. Thus the averages (25) vanish and $Q$ also vanishes.

\section{Evolution of Wigner function}

The evolution of the Wigner function is governed by the following equation:

$$
\frac{\partial W}{\partial t}=\kappa\left[\frac{\partial}{\partial \alpha} \alpha+\frac{\partial}{\partial \alpha^{*}} \alpha^{*}+\frac{\partial^{2}}{\partial \alpha \partial \alpha^{*}}\right] W\left(\alpha, \alpha^{*}\right) .
$$

The solution can be written as

$$
\begin{aligned}
W\left(\alpha, \alpha^{*}, t\right)= & \frac{2}{\pi\left(1-e^{-2 \kappa t}\right)} \int d^{2} \alpha_{0} W\left(\alpha_{0}, \alpha_{0}^{*}, 0\right) \\
& \times \exp \left\{-2 \frac{\left|\alpha-\alpha_{0} e^{-\kappa t}\right|^{2}}{\left(1-e^{-2 \kappa t}\right)}\right\},
\end{aligned}
$$

where $W\left(\alpha_{0}, \alpha_{0}^{*}, 0\right)$ is the Wigner function of the initial state. It is easy to verify this solution putting Eq. (27) in Eq. (26). The time-evolution of the Wigner function of the squeezed vacuum state $|\psi\rangle$ can be easily calculated analytically using the following integral identity $[18,19]$ :

$$
\begin{gathered}
\int d^{2} \alpha \exp \left[-|\alpha|^{2}\right] \exp \left(-\frac{\mu}{\tau} \alpha^{2}-\frac{\nu}{\tau} \alpha^{* 2}-\frac{z^{*} \alpha}{\sqrt{\tau}}+\frac{z \alpha^{*}}{\sqrt{\tau}}\right) \\
=\frac{\pi \tau}{\sqrt{\tau^{2}-4 \mu \nu}} \exp \left(-\frac{\mu z^{2}+\nu z^{* 2}+\tau|z|^{2}}{\tau^{2}-4 \mu \nu}\right) .
\end{gathered}
$$

Using Eq. (13) and the above identity in Eq. (27), we get the following:

$$
\begin{aligned}
W\left(\alpha, \alpha^{*}, t\right)= & \frac{4}{\pi\left(1-e^{-2 \kappa t}\right)} \frac{\exp \left[-\frac{2|\alpha|^{2}}{1-e^{-2 \kappa t}}\right]}{\sqrt{a^{2}-4|c|^{2}}} \\
& \times \exp \left[\frac{b^{2} c^{*}+b^{* 2} c+a|b|^{2}}{a^{2}-4|c|^{2}}\right],
\end{aligned}
$$

where

$$
\begin{gathered}
a=2 \cosh (r)+2 \frac{e^{-2 \kappa t}}{1-e^{-2 \kappa t},} \\
b=\frac{2 \alpha^{*} e^{-\kappa t}}{1-e^{-2 \kappa t}}, \quad c=e^{-i \theta} \sinh (r) .
\end{gathered}
$$

Clearly the Wigner function of the squeezed vacuum state is Gaussian at all times.

We now calculate the time-dependence of the Wigner function of the state $|\psi\rangle_{1}$. The initial Wigner function, as given by Eq. (14), can be rewritten as

$$
W\left(\alpha, \alpha^{*}, 0\right)=\left.\frac{2}{\pi} D\left[e^{-\lambda|\widetilde{\alpha}|^{2}}\right]\right|_{\lambda=2}, \quad D=-4 \frac{d}{d \lambda}-1 .
$$

Using Eq. (31) and (27), we can find the following expression for the Wigner function:

$$
\begin{aligned}
W\left(\alpha, \alpha^{*}, t\right)= & \left(\frac{2}{\pi}\right)^{2} \frac{1}{1-e^{-2 \kappa t}} D \int d^{2} \alpha_{0} \exp \left[-\lambda\left|\widetilde{\alpha}_{0}\right|^{2}\right] \\
& \times\left.\exp \left[-2 \frac{\left|\alpha-\alpha_{0} e^{-\kappa t \mid}\right|^{2}}{1-e^{-2 \kappa t}}\right]\right|_{\lambda=2},
\end{aligned}
$$

where $\widetilde{\alpha}_{0}=\alpha_{0} \cosh (r)-\alpha_{0}^{*} e^{i \theta} \sinh (r)$. Simplifying the above expression using Eq. (28), we get

$$
\begin{aligned}
W\left(\alpha, \alpha^{*}, t\right)= & \frac{32 P}{\pi\left(1-e^{-2 \kappa t}\right)} \frac{\exp \left[-\frac{2|\alpha|^{2}}{1-e^{-2 \kappa t}}\right]}{\left(a^{2}-4|c|^{2}\right)^{5 / 2}} \\
& \times \exp \left[\frac{b^{2} c^{*}+b^{* 2} c+a|b|^{2}}{a^{2}-4|c|^{2}}\right],
\end{aligned}
$$

where

$$
\begin{aligned}
P= & \left(1-x^{2}\right)\left\{\sinh (2 r)\left(e^{i \theta} b^{2}+e^{-i \theta} b^{* 2}\right)\right. \\
& \left.+2\left[(x+1)^{2}+4 x \sinh ^{2}(r)\right]\right\}+2\left\{\left(1+x^{2}\right) \cosh (2 r)+2 x\right\}|b|^{2}
\end{aligned}
$$

and 


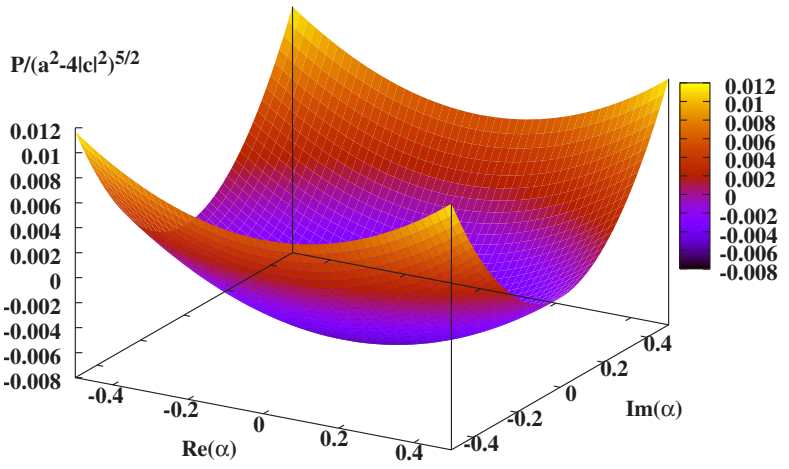

FIG. 6. (Color online) Variation of $P /\left(a^{2}-4|c|^{2}\right)^{5 / 2}$ in phase space for $r=0.31, \theta=0$, and $\kappa t=0.1$.

$$
x=\frac{e^{-2 \kappa t}}{1-e^{-2 \kappa t}} .
$$

Clearly, the Wigner function is non-Gaussian due to the presence of the polynomial $P$. This becomes negative when the polynomial $P$ becomes negative. In Fig. 6, we have plotted $C^{\prime}\left(\alpha, \alpha^{*}, t\right)=P /\left(a^{2}-4|c|^{2}\right)^{5 / 2}$ in phase space to show the negative region for the Wigner function.

Note that at the center of the phase space $\left(\alpha=\alpha^{*}=0\right)$, the Wigner function is maximally negative. At the center,

$$
C^{\prime}(0,0, t)=\frac{2\left(1-x^{2}\right)\left\{(x+1)^{2}+4 x \sinh ^{2}(r)\right\}}{\left(a^{2}-4|c|^{2}\right)^{5 / 2}}
$$

which becomes negative when $\left(1-x^{2}\right)$ becomes negative. This leads to the following condition:

(i)

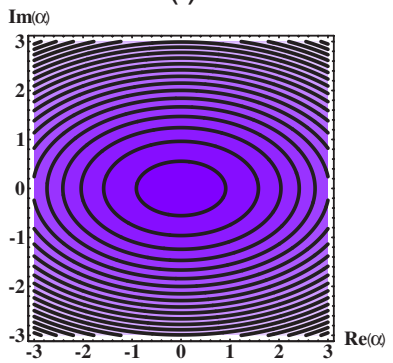

(ii)
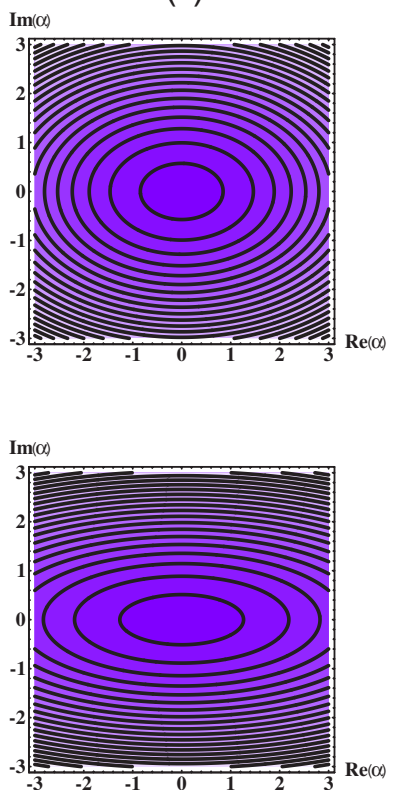

$$
\kappa t<\kappa t_{0}=\frac{1}{2} \ln (2),
$$

which is independent of the squeezing parameter $r$. Thus the Wigner function has a certain negative region for the time $t<t_{0}=\ln (2) / 2 \kappa$. However, the negative value does depend on the squeezing parameter.

We find from Eq. (34) that at $t=t_{0}$ (i.e., when $x^{2}=1$ ), $P$ becomes a circle in phase space. Thus beyond $t>t_{0}$, the ellipse $16 C^{\prime} /\left(1-e^{-2 \kappa t}\right)=$ const interchanges its minor and major axes. We show this behavior in Fig. 7 for different values of $r$. Note that at times much larger than decoherence time scale $1 / \kappa$ (i.e., for $\kappa t \rightarrow \infty$ ), $P \rightarrow 2$ and thus becomes constant throughout the phase space.

Using Eq. (33), we show the variation of Wigner function at different time scales in Fig. 8. It is easy to see how the negative region of the Wigner function gradually diminishes. At long times $\kappa t \rightarrow \infty$, the Wigner function becomes

$$
W\left(\alpha, \alpha^{*}, \infty\right)=\frac{2}{\pi} e^{-2|\alpha|^{2}},
$$

which corresponds to vacuum state. We have shown this in Fig. 1(b). This can also be understood from Eq. (23). For $\kappa t \rightarrow \infty, \rho_{0,0}$ approaches unity, whereas all other density matrix elements vanish. This means that at long times, the state decays to vacuum, as we have discussed earlier.

We next study the time-evolution of the Wigner function for the case of large squeezing, i.e., large values of $\zeta$. In this case the single photon subtracted squeezed state becomes similar to a Schrodinger cat state. For large times, such an optical cat state decays to vacuum. Thus the Wigner function becomes Gaussian, as discussed above. We show this evolution for large squeezing in Fig. 9.

(iii)
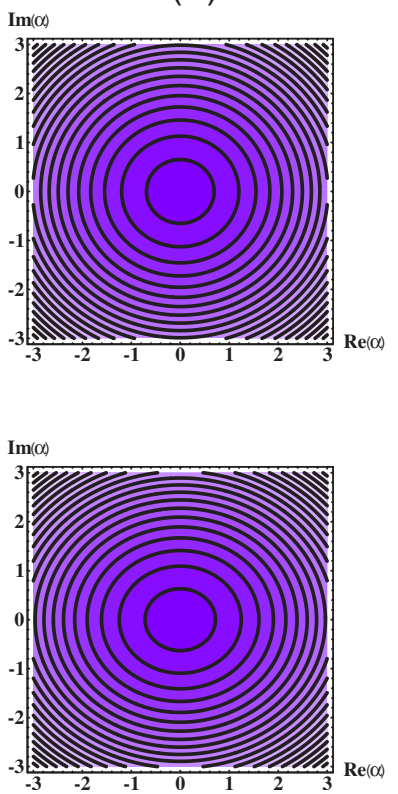

(iv)
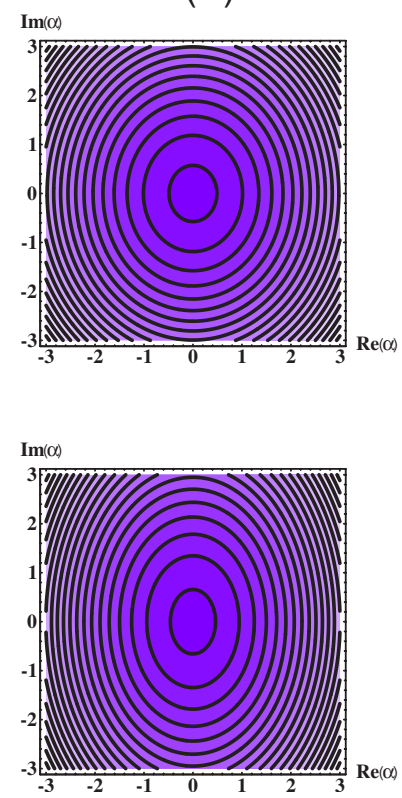

FIG. 7. (Color online) Plot of $16 C^{\prime} /\left(1-e^{-2 \kappa t}\right)$ in phase space for (a) $r=0.31$ and (b) $r=0.8$ at times (i) $\kappa t=0.05$, (ii) $\kappa t=0.1$, (iii) $\kappa t$ $=0.3$, and (iv) $\kappa t=0.5$. 
(a)

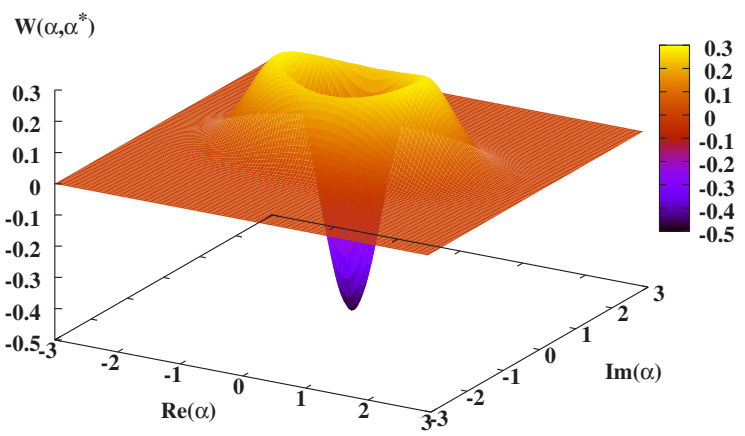

(c)

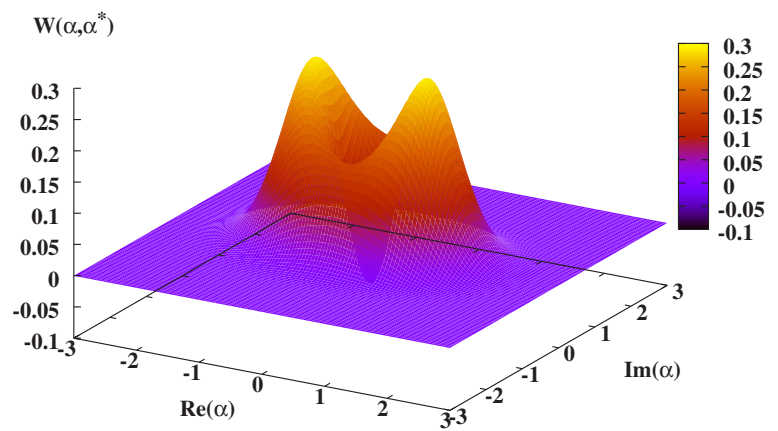

(b)

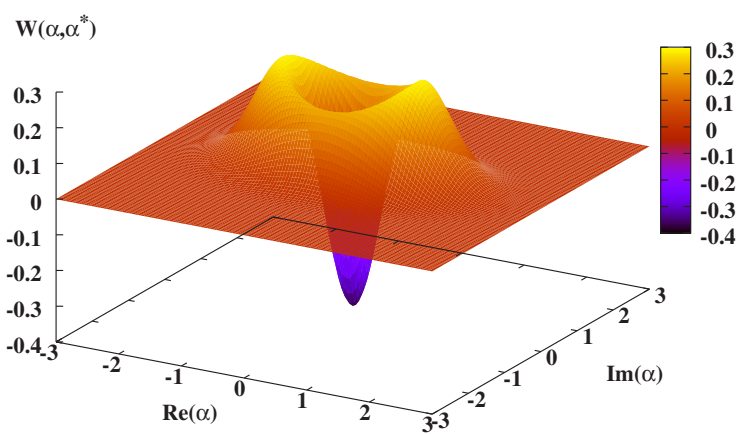

(d)

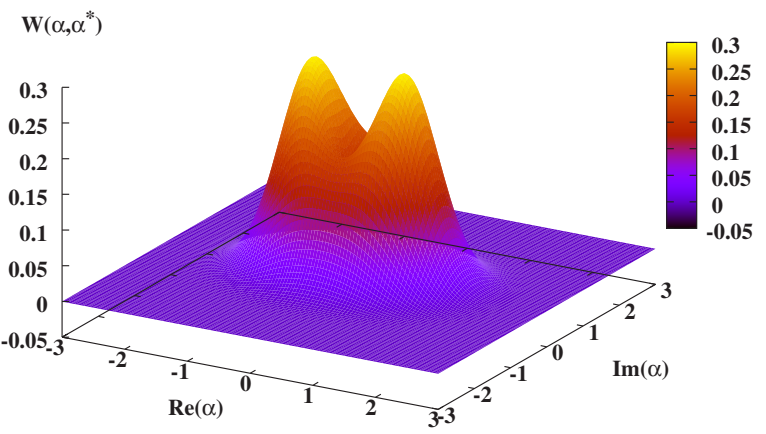

FIG. 8. (Color online) Wigner function of single-photon subtracted squeezed states for $\theta=0$ and $r=0.31$ at (a) $\kappa t=0.05$, (b) $\kappa t=0.1$, (c) $\kappa t=0.3$, and (d) $\kappa t=0.5$.

\section{B. Effect of phase damping}

We now study the effect of phase damping on the state $|\psi\rangle_{1}$. Such damping can be described by the following master equation:

$$
\dot{\rho}=-\kappa_{p}\left(A^{\dagger} A \rho-2 A \rho A^{\dagger}+\rho A^{\dagger} A\right),
$$

where $A=a^{\dagger} a$ is the number operator and $\kappa_{p}$ is the corresponding rate of decoherence. The solution of this equation can be easily found as Eq. (20) where

$$
\rho_{n, n^{\prime}}(t)=\exp \left[-\left(n-n^{\prime}\right)^{2} \kappa_{p} t\right] \rho_{n, n^{\prime}}(0) .
$$

It is easy to see that only the diagonal elements $\rho_{n, n}$ do not decay due to dephasing. Thus at long times, we can write

$$
\rho(t \rightarrow \infty)=\sum_{n=0}^{\infty} \rho_{n, n}(0)|n\rangle\langle n|,
$$

which refers to a mixed state.

Using Eqs. (25) and (40), we next calculate the parameter $Q$. We find that the averages $\left\langle a^{\dagger 2} a^{2}\right\rangle$ and $\left\langle a^{\dagger} a\right\rangle$ do not depend upon time, because in the case of phase damping $\rho_{n, n}(t)$ $=\rho_{n, n}(0)$. Thus $Q$ remains the same for all times. However, the corresponding Wigner function has certain timedependence. We find that at long times, the Wigner function becomes

$$
W\left(\alpha, \alpha^{*}, \infty\right)=\sum_{n=0}^{\infty} \rho_{n, n}(0) W_{|n\rangle\langle n|}\left(\alpha, \alpha^{*}\right),
$$

where $W_{|n\rangle\langle n|}\left(\alpha, \alpha^{*}\right)$ is the Wigner function of a Fock state $|n\rangle$ as given by

$$
W_{|n\rangle\langle n|}\left(\alpha, \alpha^{*}\right)=(-1)^{n} \frac{2}{\pi} e^{-2|\alpha|^{2}} L_{n}\left(4|\alpha|^{2}\right) .
$$

The function (42) refers to a highly nonclassical state. It is interesting to note that all the Fock states have independent contributions to the Wigner function at long times, weighted by their initial population $\rho_{n, n}(0)$. On the other hand, in the case of decoherence due to photon-number decay, only the vacuum state survives. In Fig. 10, we plot the Wigner function (42) in phase space for different squeezing. Note that the Wigner function has a negative region at long times representing nonclassicality for all $r$, even if the state does not exhibit sub-Poissonian statistics for $r \gtrsim 0.46$ (because $Q$ is positive). In fact, if $Q$ is positive, it does not mean that the state is classical. In such cases, we have to use other parameters to test the nonclassicality. Several parameters have been introduced in this context $[16,20]$. We can use hierarchy of these parameters which have been shown to be especially useful in the context of cat states. Here we illustrate the utility of one such parameter, e.g., the $A_{3}$ parameter as defined by [16] 
(a)

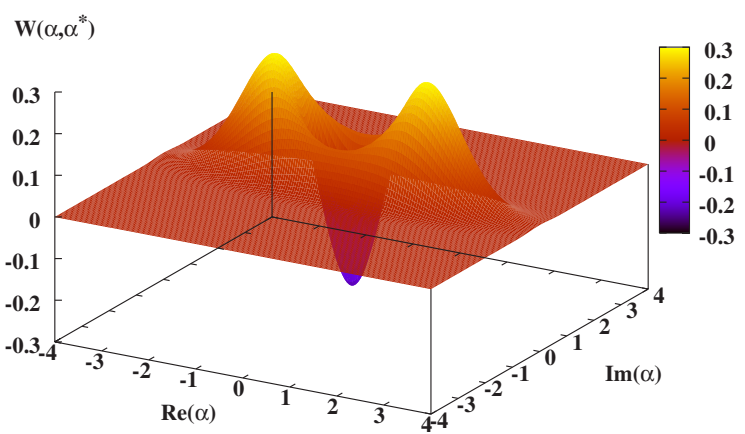

(c)

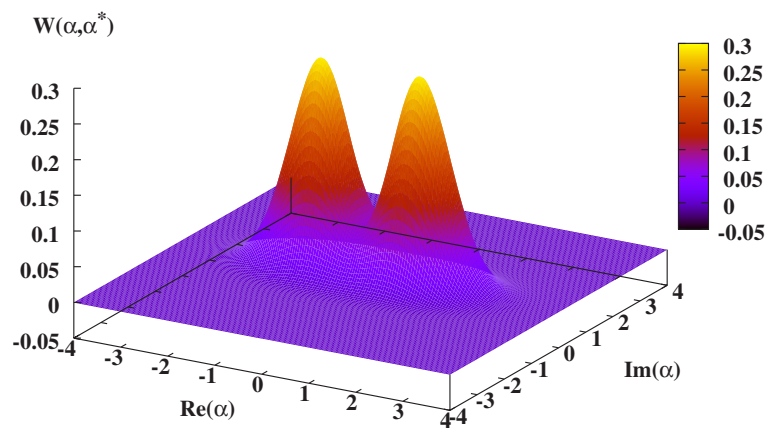

(b)

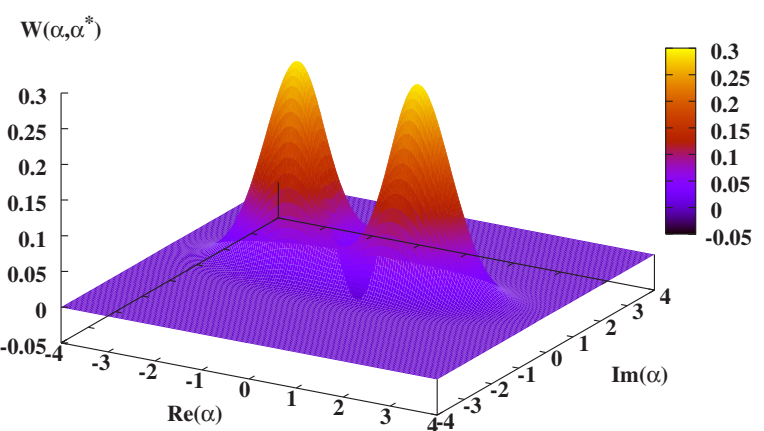

(d)

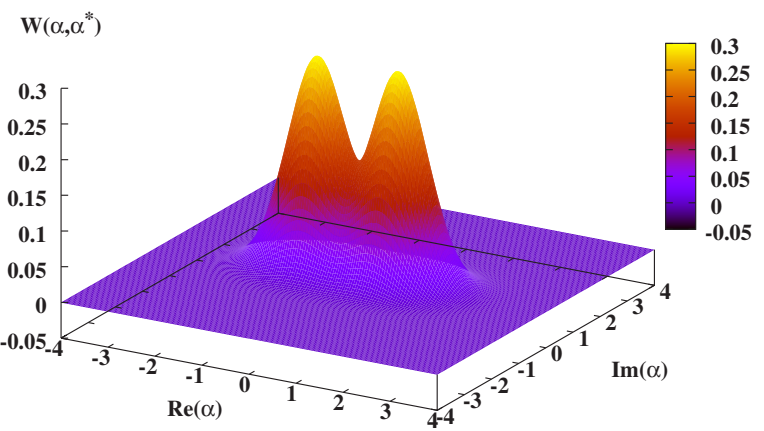

FIG. 9. (Color online) Wigner function of single-photon squeezed states for $\theta=0$ and $r=0.8$ at (a) $\kappa t=0.1$, (b) $\kappa t=0.3$, (c) $\kappa t=0.5$, and (d) $\kappa t=0.7$.

$$
A_{3}=\frac{\operatorname{det}\left[m^{(3)}\right]}{\operatorname{det}\left[\mu^{(3)}\right]-\operatorname{det}\left[m^{(3)}\right]},
$$

where

$$
m^{(3)}=\left(\begin{array}{ccc}
1 & m_{1} & m_{2} \\
m_{1} & m_{2} & m_{3} \\
m_{2} & m_{3} & m_{4}
\end{array}\right), \quad \mu^{(3)}=\left(\begin{array}{ccc}
1 & \mu_{1} & \mu_{2} \\
\mu_{1} & \mu_{2} & \mu_{3} \\
\mu_{2} & \mu_{3} & \mu_{4}
\end{array}\right),
$$

$m_{s}=\left\langle a^{\dagger s} a^{s}\right\rangle, \mu_{s}=\left\langle\left(a^{\dagger} a\right)^{s}\right\rangle$ and det indicates determinant of the matrix. The state exhibits phase-insensitive nonclassical

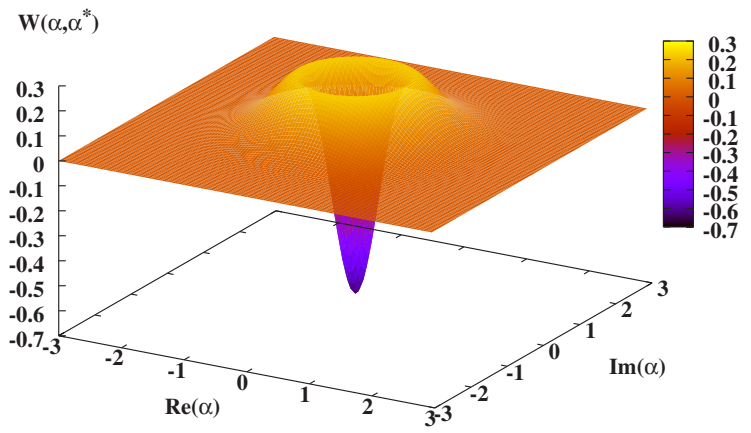

(a) properties if $A_{3}$ lies between 0 and -1 [16]. For the state $|\psi\rangle_{1}$ we have found that $A_{3}$ remains negative for $|\xi| \lesssim 0.6$ which corresponds to $r \lesssim 0.7$. Clearly $A_{3}$ is a stronger measure of nonclassicality than $Q$ because it leads to a larger upper bound of $r$ to exhibit nonclassicality. Further, comparing the Wigner functions in Fig. 10 with those at $t=0$ (see Fig. 2), we find that the Wigner function varies very slowly with time for small squeezing; but for large squeezing, the variation is faster. We can conclude that phase damping leads to much slower decoherence than amplitude damping.

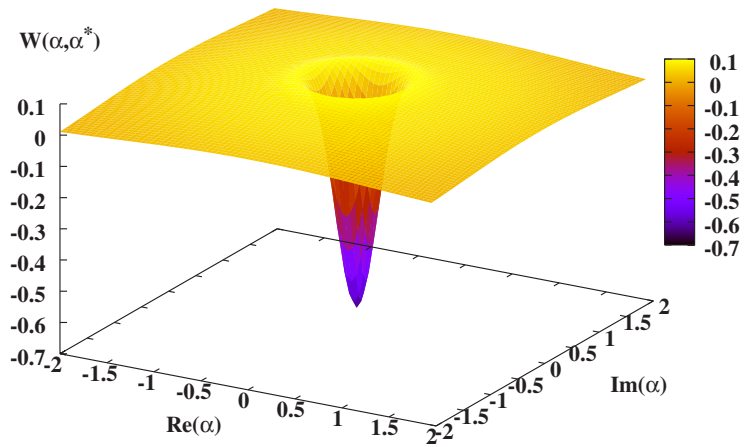

(b)

FIG. 10. (Color online) Wigner function in phase space at long times in the presence of phase damping for (a) $r=0.31$ and (b) $r=0.8$. 


\section{CONCLUSIONS}

In conclusion, we have studied how a class of nonGaussian states evolves in the presence of decoherence. We have considered a single-photon subtracted squeezed vacuum state, the Wigner function of which is similar to that of a Schrodinger kitten state. We have found an upper bound for the squeezing parameter for which this state exhibits subPoissonian photon statistics. However, the state remains nonclassical for all values of the squeezing parameter because the Wigner function becomes negative around the central region in phase space. Next, we have studied how the state evolves in the presence of two different kinds of decoherence, viz. amplitude decay and phase damping. We have found analytical expressions for the time-evolution of the state and the Wigner function in both cases. In the case of amplitude decay, the Wigner function loses its non-Gaussian nature and becomes Gaussian at long times, corresponding to vacuum. On the other hand, phase damping leads to much slower decoherence than amplitude damping. The state remains nonclassical at long times.

\section{ACKNOWLEDGMENTS}

A.B. gratefully acknowledges the partial support from the Women at the Science and Engineering program at the University of Southern California, Los Angeles, USA. G.S.A. kindly acknowledges support from NSF Grant No. CCF0524673.
[1] J. Wenger, R. Tualle-Brouri, and P. Grangier, Phys. Rev. Lett. 92, 153601 (2004).

[2] M. S. Kim, E. Park, P. L. Knight, and H. Jeong, Phys. Rev. A 71, 043805 (2005).

[3] J. Eisert, S. Scheel, and M. B. Plenio, Phys. Rev. Lett. 89, 137903 (2002); D. E. Browne, J. Eisert, S. Scheel, and M. B. Plenio, Phys. Rev. A 67, 062320 (2003).

[4] G. Giedke and J. I. Cirac, Phys. Rev. A 66, 032316 (2002).

[5] H. Nha and H. J. Carmichael, Phys. Rev. Lett. 93, 020401 (2004); R. Garcia-Patron, J. Fiurasek, N. J. Cerf, J. Wenger, R. Tualle-Brouri, and P. Grangier, ibid. 93, 130409 (2004).

[6] S. D. Bartlett and B. C. Sanders, Phys. Rev. A 65, 042304 (2002).

[7] A. Ourjoumtsev, R. Tualle-Brouri, J. Laurat, and P. Grangier, Science 312, 83 (2006).

[8] J. S. Neergaard-Nielsen, B. M. Nielsen, C. Hettich, K. Mølmer, and E. S. Polzik, Phys. Rev. Lett. 97, 083604 (2006).

[9] V. Buzek and P. L. Knight, Progress in Optics, edited by E. Wolf (North Holland, Amsterdam, 1195), Vol. XXXIV, p. 1.

[10] J. Fiurasek, R. Garcia-Patron, and N. J. Cerf, Phys. Rev. A 72, 033822 (2005).
[11] G. S. Agarwal and K. Tara, Phys. Rev. A 43, 492 (1991).

[12] A. Zavatta, S. Viciani, and M. Bellini, Science 306, 660 (2004); Phys. Rev. A 72, 023820 (2005).

[13] M. Sasaki and S. Suzuki, Phys. Rev. A 73, 043807 (2006).

[14] C. Invernizzi, S. Olivares, M. G. A. Paris, and K. Banaszek, Phys. Rev. A 72, 042105 (2005); S. Olivares and M. G. A. Paris, ibid. 70, 032112 (2004); S. Olivares, M. G. A. Paris, and R. Bonifacio, ibid. 67, 032314 (2003).

[15] L. Mandel, Opt. Lett. 4, 205 (1979).

[16] G. S. Agarwal and K. Tara, Phys. Rev. A 46, 485 (1992).

[17] G. S. Agarwal, Progress in Optics, edited by E. Wolf (North Holland, Amsterdam, 1973), Vol. XI, p 1.

[18] G. S. Agarwal and E. Wolf, Phys. Rev. D 2, 2187 (1970), Eq. (6.15); V. Bargmann, Commun. Pure Appl. Math. 14, 187 (1961).

[19] R. R. Puri, Mathematical Methods of Quantum Optics (Springer-Verlag, Berlin, 2001), Appendix A.

[20] G. S. Agarwal, Opt. Commun. 93, 109 (1993); W. Vogel, Phys. Rev. Lett. 84, 1849 (2000); Th. Richter and W. Vogel, ibid. 89, 283601 (2002). 